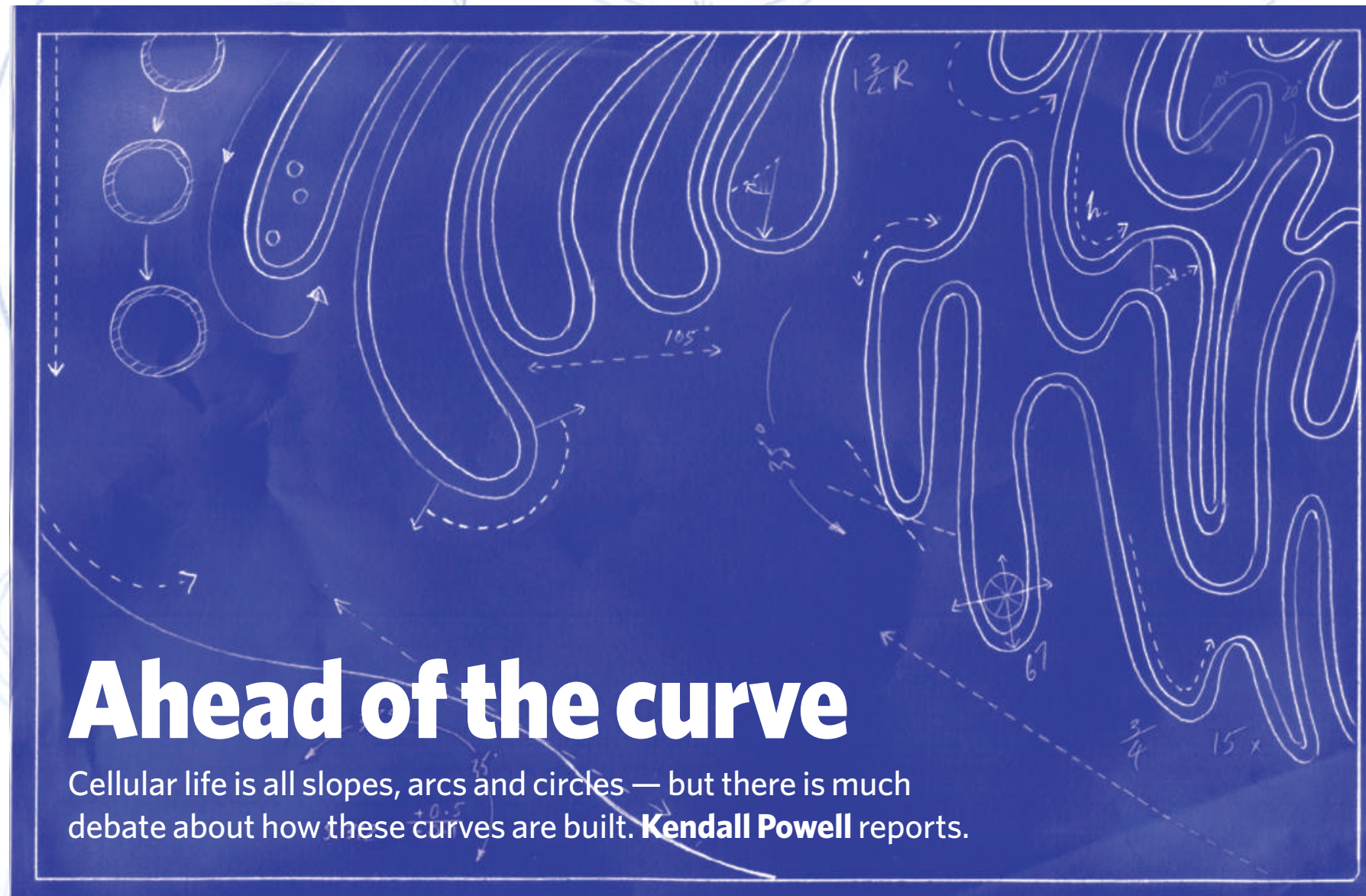

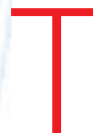

o view the innards of a cell is to view architecture reminiscent of Antoni Gaudi: the gentle arc of the cell membrane, the contortions of internal tubing, the tight bubbles of vesicles. But for biologists, this architecture is an intellectual puzzle as well as a beautiful structure. Membranes generally prefer to be flat - so what, exactly, is generating all the curves?

Pietro De Camilli thought he had found one answer when, just over a decade ago, he saw a tangle of tubules in an electron micrograph. The pictures came from an experiment carried out by Kohji Takei, De Camilli’s postdoc at Yale University in New Haven, Connecticut. Takei had mixed a protein called amphiphysin, which is thickly clustered at the tips of neurons, with large bubbles of artificial membranes to try to replay the process by which neurotransmitter chemicals are packaged up. It came as a surprise when the membranes snapped into masses of jumbled, twisted tubes ${ }^{1}$. "It looked like a plate of spaghetti, it was absolutely spectacular," says De Camilli. "It was the first example of a protein that, by itself, had dramatic membranedeforming properties," he says.

The first, maybe - but not the last. Since this discovery, De Camilli and others have identified whole families of protein that have equally dramatic abilities to bend membranes, and a tightly knit community of researchers has built up to study them. Much debate has centred on whether the proteins create curves by wedging themselves into membranes, or by moulding them into shape using curved protein scaffolds. As it turns out, both may be at play - and more.

The issue is not just one of beautiful architecture: the ability to turn membranes into circles and tubes is central to almost every cellular process. Spherical vesicles are essential for carrying construction materials and communication signals around the cell. And the undulating network of membranes that makes up the mitochondria, endoplasmic reticulum (ER) and Golgi complex are vital to these organelles' roles in energy production, protein synthesis and protein processing, respectively. Some researchers are now finding that, once built, the cell

"It looked like a
plate of spaghetti,
it was absolutely
spectacular."
- Pietro De Camilli

Laboratory of Molecular Biology in Cambridge, $\frac{\mathrm{\alpha}}{\widetilde{\alpha}}$ UK. "Evolution from the primordial singlecelled organisms to multicellular organisms was made possible by the appearance of small membrane-bound spaces in the cell," he says.

\section{A matter of scale}

The closest that most cells come to having straight lines are the surfaces of the encircling plasma membrane. On tightly packed and column-shaped cells, these are virtually flat; and even on curved cells, a particular spot on the circumference still seems level to the molecules there, much like the surface of Earth does to a person standing on it. Inside the cell, however, microscopy has revealed a landscape with uses its curves to position cellular processes. "The fact that the shape can also be information is very exciting," says Bruno Antonny, a biochemist at the CNRS Institute of Molecular and Cellular Pharmacology in Valbonne, France. "That you can sense curvature means that you can organize reactions in time and space."

Walling off spaces by wrapping membranes around them was also necessary for the evolution of cellular and organismal complexity, as it allowed cells to adopt specialized functions and trafficking to occur between compartments the hallmarks of eukaryotic cells, says Harvey McMahon of the Medical Research Council much sharper contours. Yet for many years researchers gave little thought to how such curves were generated: cell membranes were thought of as fluid, pliable structures that could easily be pushed or pulled into shape.

That view changed in the early 1970s when physicist Wolfgang Helfrich, one of those behind the invention of the liquid-crystal display, proposed a model showing that membranes are relatively rigid structures that require substantial energy to contort ${ }^{2}$, just as a liquid crystal does. Cell membranes are made up of lipid bilayers: two tightly packed rows of lipid molecules lined up with their hydrophilic lipid head 
groups facing outwards, and water-repellent fatty-acid chains sandwiched on the inside. Work by Helfrich and other biophysicists has since shown that lipids prefer to be lined up in planes, and it takes a lot of energy to create the kind of disturbance that comes with bending a plane into a cylinder or sphere. The situation is similar to blowing a soap bubble from a circular wand - it takes continuous blowing to deform the flat soap film into a sphere.

Researchers spent many years looking for scaffolding proteins that could sustain a membrane curve, but none emerged that could, according to biophysicists' calculations, provide sufficient energy. De Camilli says that it wasn't until the 1999 amphiphysin experiment that biologists began to appreciate that proteins could be 'massaging' membranes into shape rather than forcing them. What made that discovery all the more intriguing to De Camilli was that amphiphysin is normally found floating in the cell cytosol, not integrated into membranes. He wondered whether there were more proteins that could distort membranes so strongly, and how they did it.

By 2002, De Camilli's group and others had identified a few more proteins that had membrane-flexing abilities ${ }^{3-5}$ - and saw that they shared an intriguing spiral structure called an amphipathic helix. Looking down the barrel of the helix, one half is charged and the other half uncharged. Put the protein near a bilayer and it will immediately insert itself lengthwise with its charged half among the lipid head groups and the uncharged half nestled within the fatty-acid chains. This suggested that insertion into the outer half of the bilayer would create a wedge, and if enough wedges were inserted then the membrane would bend (see graphic, overleaf). It was an instantly appealing idea and one that De Camilli promoted - but it was about to be joined by an equally appealing rival.

In 2004, a group led by McMahon

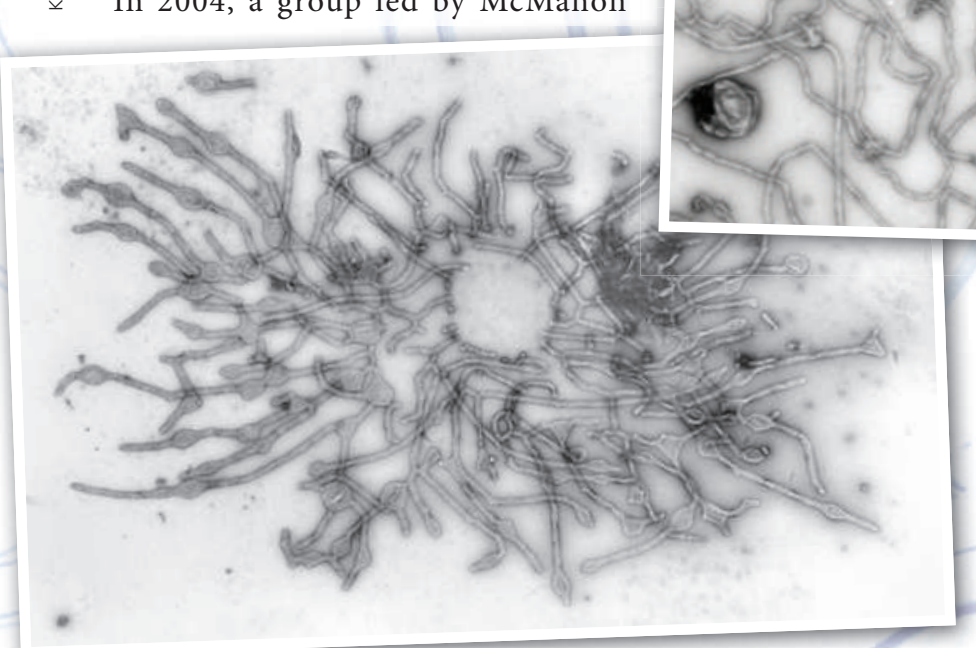
their own curved shape.

and his colleague Phil Evans solved the three-dimensional structure of the 'BAR' domain of amphiphysin, which includes the amphipathic helix ${ }^{6}$. The helix didn't crystallize well, but the rest of the BAR domain did - and their work showed that it was crescent shaped. "We could immediately see this banana shape, that, to me, is one of the most beautiful structures we've solved," McMahon says. Beauty aside, the banana suggested an alternative way for the BAR domain to bend membranes. The concave face of the banana is positively charged, which would make it stick to the negatively charged outer surface of membranes and mould them to

The bananas did not go down well at meetings, and McMahon recalls more than a few scoffs and scowls. The idea that they were acting as scaffolding seemed too similar to previous scaffolding models, which the physicists had already proved

"We could immediately
see this banana shape,
that, to me, is one of the
most beautiful structures
we've solved."
$\quad$ - Harvey McMahon
wrong. Clearly BAR domains were important: evidence was piling up that they were a huge family and the various relatives could subject membranes to all sorts of bending. But were the banana-shaped molecules generating the curvature or simply stabilizing or sensing the curvature after it had been produced by insertion of the amphipathic helix?

The question has inspired vigorous debate and some flip-flopping between ideas. Although De Camilli says he was originally sceptical of the scaffolding concept, his work now

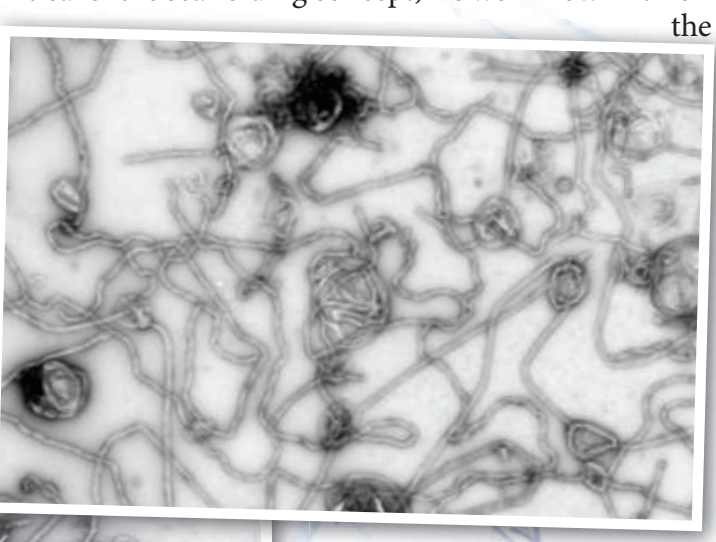

promotes it. Last year, his group, in collaboration with Yale colleague Vinzenz Unger, published stunning cryo-electron microscopy images of BAR domains without amphipathic helices. The domains stacked up end to end to form a spiral around membrane cylinders much like a barber's pole, suggesting that scaffolding alone can generate curvature - at least at the artificially high concentrations present in the test tube.

\section{Rods and curves}

McMahon now raises an eyebrow at De Camilli's latest model for how thousands of banana domains could generate curvature alone. Work from his lab has shown that this domain is much less efficient at forming membrane tubes in the lab dish if the amphipathic helix has been lopped off. "This was the first demonstration that the two functions might be joined in one protein," says McMahon.

Recently, McMahon has started to promote the wedging idea. He teamed up with biophysicist Michael Kozlov at Tel Aviv University in Israel, who calculated that insertion of an amphipathic helix alone is energetically sufficient to generate curves, whereas scaffolding alone is not. The study showed that the most efficient way to bend a membrane is to insert a short rod shape, similar to the amphipathic helix, into the outer layer of the membrane at a depth - and this is key - of around one-third of the membrane's thickness ${ }^{8}$. Their calculations predicted that the bending ability of BAR domains can be credited solely to the insertion of the amphipathic helix. "Now the feeling is that this banana-like shape stabilizes the curvature that is generated by some sort of insertion into the membrane," says Kozlov. Speaking of McMahon, De Camilli says, "Both of us got it partially right and partially wrong, so it has been a humbling experience."

Just as researchers were finding something to agree on about BAR domains, a whole new hypothesis for generating curves burst onto the scene. This one came from the lab of

Oodles of tubules: amphiphysin triggers cell membranes to assemble into tubes.
Tom Rapoport at Harvard Medical School in Boston, Massachusetts. In the late 1990s, his graduate student Lars Dreier was mixing nuclear-envelope membranes with DNA, attempting to imitate in a test tube the way that the envelope reforms after cell division. In the background of the microscope slide, Dreier noticed a network of membrane tubules forming that closely resembled the ER, the main site of protein synthesis in the cell. When 
Rapoport saw it, he says, "I ran around the lab asking everyone, 'Did you see this? It's fantastic!' and getting everyone to look through the microscope". The experiment seemed to have triggered membranes into self-assembling an $\mathrm{ER}$, which is normally continuous with the nuclear envelope membrane ${ }^{9}$.

The excitement only intensified when Gia Voeltz, then one of Rapoport's postdocs, isolated a class of proteins responsible for curving the tubules in the $\mathrm{ER}^{10}$. Called reticulons, these proteins have neither a BAR domain nor an amphipathic helix and there is much discussion as to how they work. They do form a double hairpin-loop structure that inserts partway into the membrane, and Rapoport and Voeltz, along with De Camilli and Kozlov, think that these hairpins may act as a wedge. (Kozlov has calculated that the hairpins probably insert to the magical one-third depth.)

\section{The barber pole effect}

Because reticulons are sunk permanently into the membrane, Voeltz, who is now at the University of Colorado, Boulder, says that they and proteins like them might be better suited for the long-term maintenance of an organelle's tubular shape than a protein such as amphiphysin, which inserts temporarily as a budding vesicle forms. She originally thought that the reticulons might stack up to make a "nice little barber pole" scaffold around the ER tubes. But now, she's pretty sure it's not that simple - and when she pulls up her lab's latest cryo-electron microscopy images of the ER tubes, it's easy to see why.

In these images, which show the ER's threedimensional structure at nanometre-scale, the tubules look nothing like neat cylinders and more like gnarly tree roots with wide and narrow stretches. Voeltz now thinks that a clus-

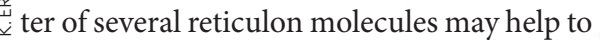
stabilize the tubes by forming a half-ring at the narrowest points of constriction. This fits

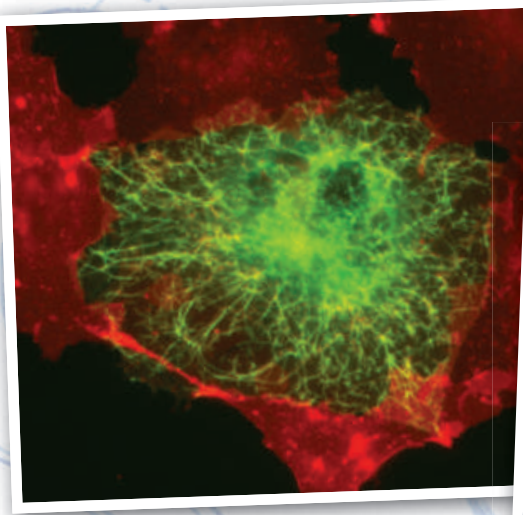

BAR domains conjure tubules out of cell membranes.

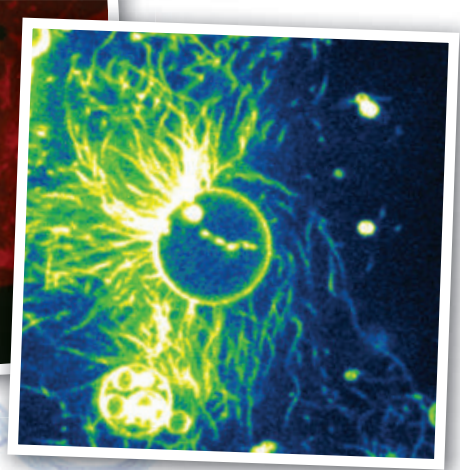

BENDING: THE RULES

Two possible ways to bend a membrane: insertion of wedges, such as amphipathic helices, or moulding around a scaffold.
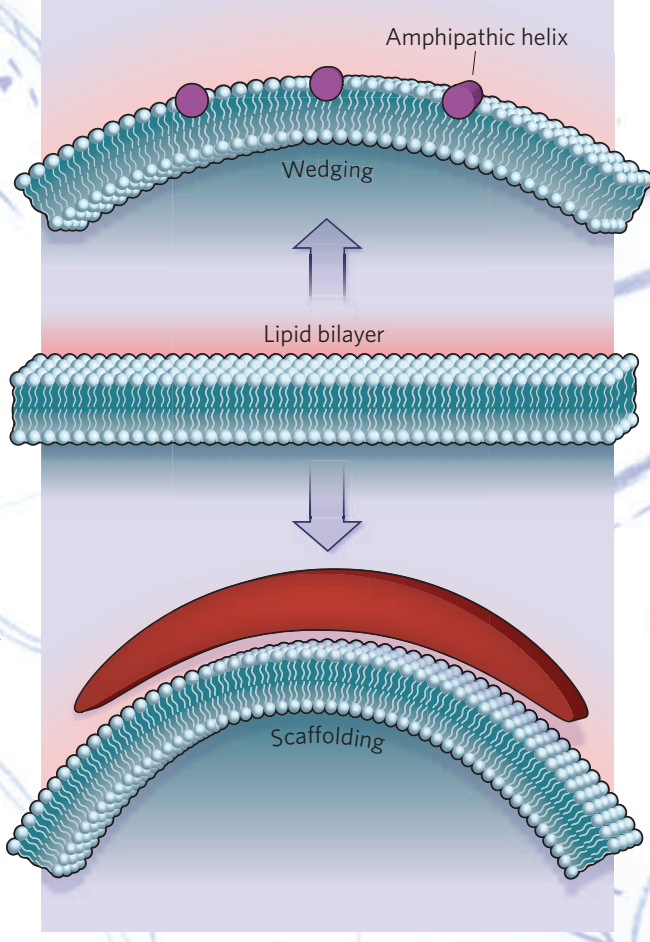

with the idea that cells have evolved multiple wedges, with different shapes and bending abilities, to fit different purposes.

McMahon now says it was clear early on, "that we were going to have a whole repertoire of proteins - some driving curvature, some limiting or stabilizing curvature, and some sensing curvature". And Antonny's group has been focused on the sensing part of the repertoire. He is trying to understand how the bend of a membrane can act as a signal, such that its position, camber or direction can recruit additional proteins to a particular point on an organelle.

Antonny's recent work has focused on one enzyme, called ArfGAP1, that detects the tight curve on transport vesicles and directs the
removal of their protein coat before they can fuse with their destination membrane. His team showed that ArfGAP1 has greater activity when bound to highly curved membrane spheres - with the same diameter as transport vesicles than to ones with a broader diameter and a gentler curve ${ }^{11}$. The degree of curvature tells the enzyme that there's a vesicle that needs uncoating, Antonny says, so that it binds only to vesicles and not to other curved surfaces.

By chopping ArfGAP1 into smaller bits, the group found that its key curve-sensing stretch was an amphipathic helix. "But the chemistry of the helix was almost the opposite of what you would expect," he says. Unlike that in the BAR domain, the helix in ArfGAP1 has almost no positive charge on one side and lacks the strong attraction to the membrane ${ }^{12}$. Antonny compares the protein's behaviour to that of a nervous swimmer about to take the plunge. "It's as if the molecule is shy and the water is not warm enough when the membrane is flat. But when you bend the membrane, the molecule can sense the lipid packing defect and inserts." The molecule's shyness is what makes it a good sensor, he explains. It dives in only when the amount of curvature is right and gets out when that curvature changes.

Last year, Antonny's lab found a similar curvature-sensing helix that may help to maintain the shape of the Golgi complex ${ }^{13}$, an organelle responsible for processing and trafficking proteins and other large molecules in the cell. The Golgi is a series of flattened membrane sacs stacked up like pancakes, with transport vesicles constantly budding off from its fringes. How it maintains its architecture in the midst of all this trafficking has been a puzzle. The new helix "is a start to explaining why the Golgi has this beautiful architecture", says Antonny.

Explaining beautiful - but functional architecture is after all what the whole field is about. "They strike us, they surprise us, but we find a remarkably appealing harmony in such buildings," says De Camilli of Gaudi's constructions. And cells, he says, are the same. "I think the beauty of great architecture, like the beauty of cellular structures, resonates in us precisely because they build on natural physical principles."

Kendall Powell is a freelance science writer based in Broomfield, Colorado.

\footnotetext{
1. Takei, K., Slepnev, V. I., Haucke, V. \& De Camilli, P. Nature Cell Biol. 1, 33-39 (1999).

2. Helfrich, W. Z Naturforsch C 28, 693-703 (1973)

Farsad K. et al. J. Cell Biol. 155, 193-200 (2001).

4. Ford, M. G. J. et al. Nature 419, 361-366 (2002)

Antonny, B. Curr. Opin. Cell Biol. 18, 386-394 (2006)

6. Peter, B. J. et al. Science 303, 495-499 (2004).

7. Frost, A. et al. Cell 132, 807-817 (2008)

8. Campelo, F., McMahon, H. T. \& Kozlov, M. M. Biophys. J. 95, 2325-2339 (2008).

9. Dreier, L. \& Rapoport, T. A. J. Cell Biol. 148, 883-898 (2000)

10. Voeltz, G. K., Prinz, W. A., Shibata, Y., Rist, J. M. \& Rapoport T. A. Cell 124, 573-586 (2006)

11. Bigay, J., Gounon, P., Robineau, S. \& Antonny, B. Nature 426 563-566 (2003).

12. Drin, G. et al. Nature Struct. Mol. Biol. 14, 138-146 (2007)

13. Drin, G. et al. Science 320, 670-673 (2008).
} 